EPJ Web of Conferences 92,02084 (2015)

DOI: $10.1051 /$ epjconf/ 20159202084

(C) Owned by the authors, published by EDP Sciences, 2015

\title{
Heat transfer in rotor stator cavity
}

\author{
Ladislav Snajdarek $^{1,}$, Jiri Pospisil ${ }^{1}$ and Marian Brazdil ${ }^{1}$ \\ ${ }^{I}$ Brno University of Technology, Department of Power Engineering, Technická 2896/2, 61669 Brno, Czech Republic
}

\begin{abstract}
This paper presents a procedure for experimental determining the local heat transfer coefficient in the rotor-stator cavity. As an operating medium is used a hot air with various mass flow rate and rotating speed of the cavity wall. The surface temperature and heat flux distribution takes into account the change of material properties as a function of thermal distribution.
\end{abstract}

\section{Introduction}

This article describes the first experimental investigation to determine heat transfer coefficient in a concentric annulus. One of the motivations for describing the heat transfer in cavities with the rotating wall is a problem of thermal expansion and displacement of the rotor and stator parts. in rotating machines. This problem is cruicial during turbines start-up from a cold-state. The cycles of metal changing lead to thermal fatigue and metal cracking The knowledge of the heat transfer and thermal distribution in rotor/stator parts of steam and gas turbine allows to predict more efficiency machines due to due to a clearance reduction like in bandage and labyrinth seals without friction between rotor/stator wall.

\section{Literature survey}

There is many papers describes the heat transfer in cavities with rotating wall. The experimental investigation of the turbine components thermal stress is described in [5.6] where the inverse method to determine the heat flux form steam to a rotor was used. The experimental investigation of the heat transfer from steam to turbine casing in a full scale $200 \mathrm{MW}$ turbine during start-up is described in [1,2]. The heat transfer coefficient was obtained by measurement of the casing wall temperature distribution. In these papers were the empirical formulas published. Model technique of the $500 \mathrm{MW}$ steam turbine casing to determine heat transfer was used by [3]. Authors creates a model from epoxy in scale 1:3 to the real parts of turbine cylinder surfaces, but in this case the influence of rotational speed was not considered. The assumption that the fluid radial velocity is invariant across the cavity axial gap was used in [4], but in many cases is this assumption may not be appropriate.

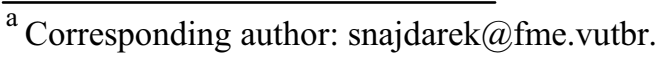

For the flow between rotating cylinder are characteristic Taylor vortices phenomena. Many research studies were published in this issue like $[7,8]$. Taylor number describes a regime of the toroid vortices in the annular gap. These vortices have effect on increasing Nusselt number.

\subsection{Labyrinth seals}

An important part of rotating machines steam, gas turbines and turbo-compressors, with the character design of concentric cylinders are labyrinth seals. The flow in the labyrinth seals is more complicated due to small clearances and many geometrical modifications like stepped, honeycomb and halo design. Taylor vortices aren't so important in this case, because of inherent vortex in labyrinth cavities.

\section{Experiments}

We considered smooth concentring cylinders geometry to obtain the first results to determine heat transfer coefficient. The geometry of the test section is adequate to the labyrinth seal ring in steam turbine with model ratio $1: 2$ but without the labyrinth. Only temperatures in stator part of the test section are measured for this time. The geometry of the test section also allows to measure heat transfer on a stator due to centrifugal forces of the rotor disc. The working fluid can be steam or air.

For the further work the correlation between steam and air will be consider. 


\subsection{Experimental apparatus}

Experimental apparatus is shown in figure 1. Hot air is used as a working fluid for these first experiments. Air is provided to the apparatus by two compressors with a maximum absolute pressure 12 bar. A stable air input to the test section is adjusted by a pressure reducing valve. The pressure is set up 1 bar. The volume mass flow rate is measured by a calorimetric meter with range $0 \div 1250$ $\mathrm{Nl} / \mathrm{min}$. Two electric heaters allow to heated air of about $170^{\circ} \mathrm{C}$ at a flow rate $500 \mathrm{Nl} / \mathrm{min}$.

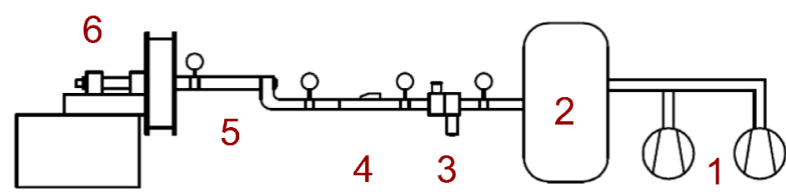

Figure 1. Experimental apparatus where 1- Two compressors, 2- Air reservoir, 3- Pressure reducing valve, 4- Calorimetric volume flow meter, 5- Two electric heaters, 6- Test section.

The test section is shown in figure 2. Hot air is supplied in the rotor/stator cavity in the axial direction. After that the air flows throw annular cylinder cavity. This cavity is shown in figure 3 . This gap width is $3.5 \mathrm{~mm}$.

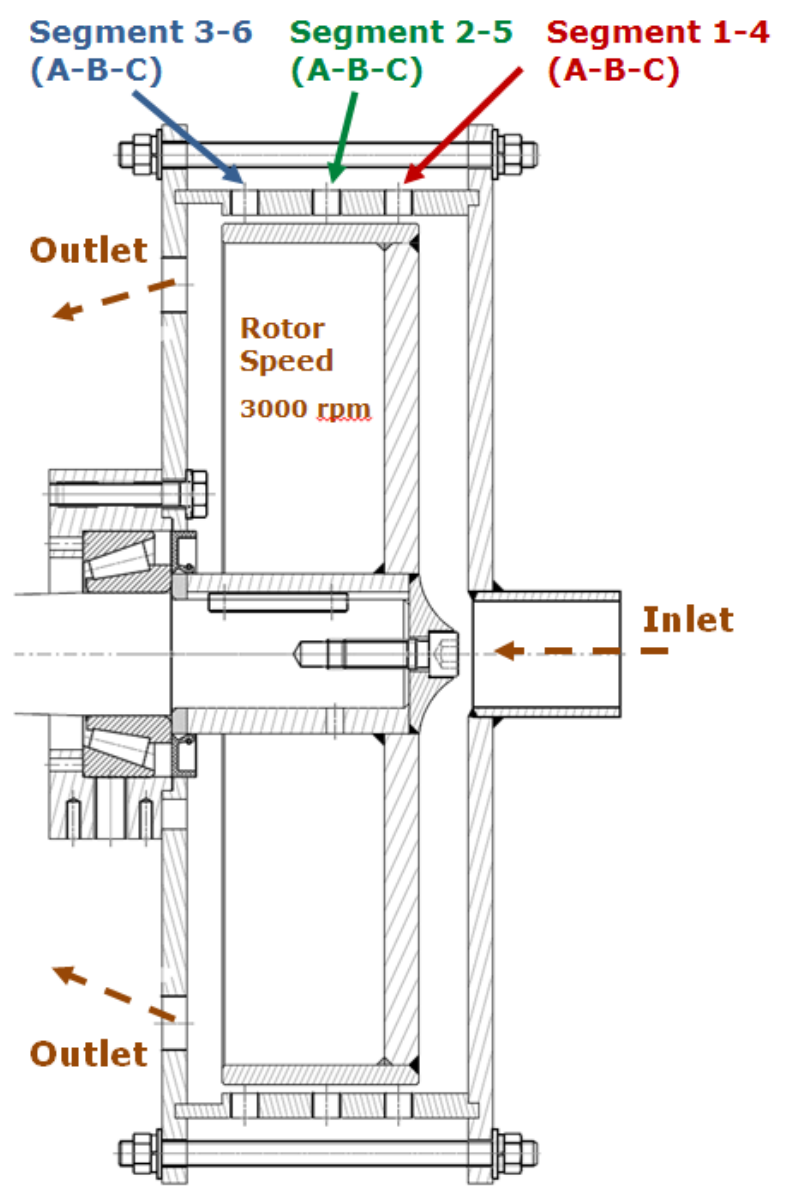

Figure 2. Test section with smooth rotor.
Rotor width is $80 \mathrm{~mm}$ and its diameter is $350 \mathrm{~mm}$. The maximum speed of the rotor is $3000 \mathrm{rpm}$ and the various speeds are allowed due to frequency inverter and measured using the eddy current sensor on the shaft. The output of the working medium is provided with three orifices having a diameter of $25 \mathrm{~mm}$.
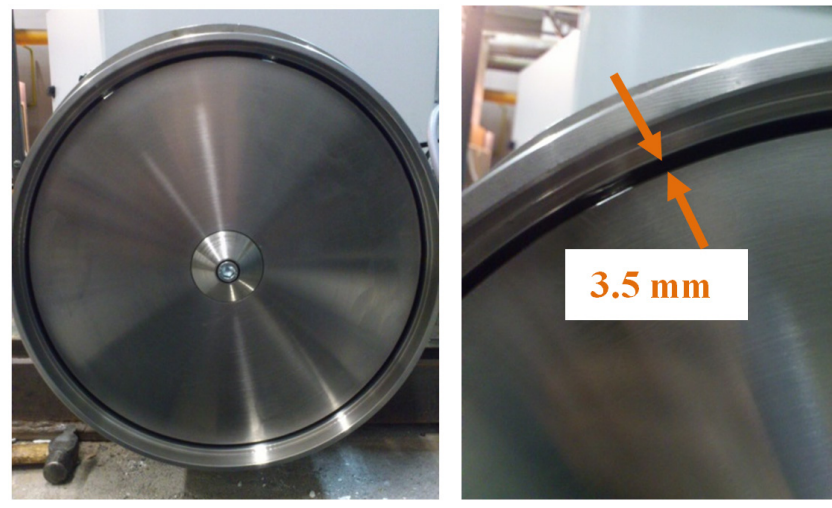

Figure 3. The annular cavity gab width.

\subsection{Measuring segments}

To evaluate the heat transfer in the cavity the system of segments are used. This system provides calibration of whole segment with three thermocouples. The concept of the temperature measuring segment is shown in figure 4.
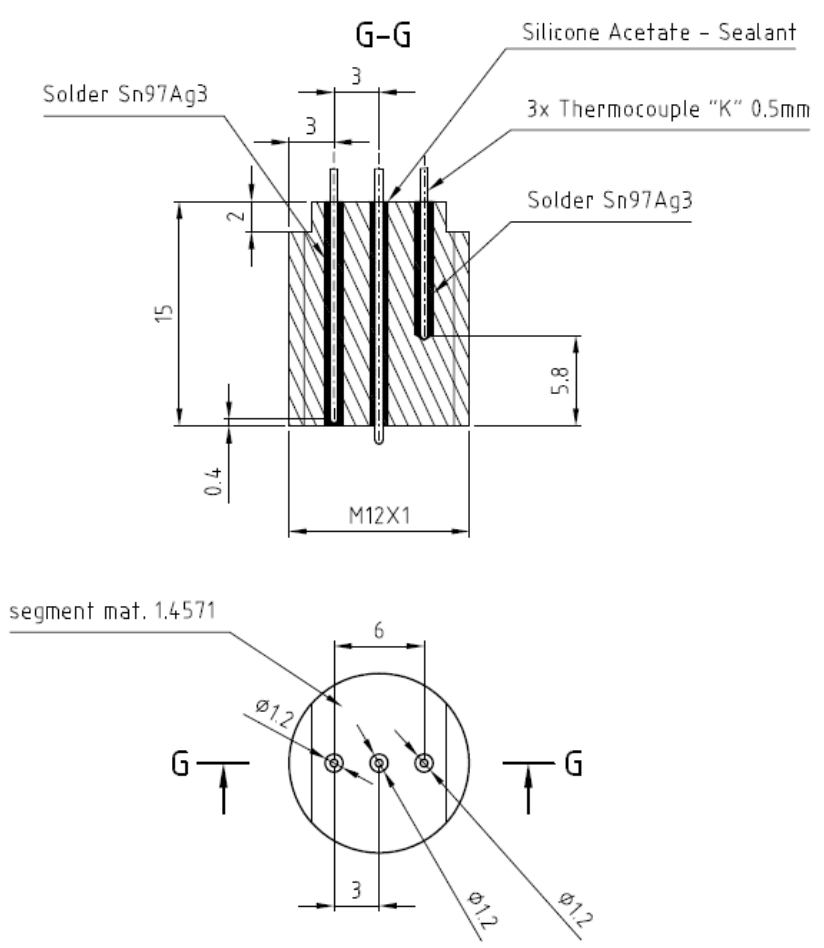

Figure 4. The temperature measuring segments.

The first thermocouple is inserted in the through hole and sealed with a solder Sn97Ag3. The distance from the stator cavity surface is $0.4 \mathrm{~mm}$ (maximum close to the 
target surface). The thermocouple is marked with the letter A for each segment.

The second thermocouple is measuring end set to a distance of $5.8 \mathrm{~mm}$ from the reference surface. The thermocouple is marked with the letter B for each segment.

The third thermocouple is ejected through holes $1 \mathrm{~mm}$ from the reference surface with respect to the environment is established and insulated silicone acrylic sealer. The thermocouple is marked with the letter $\mathrm{C}$ for each segment. The position of the segments is shown in Figure 5.
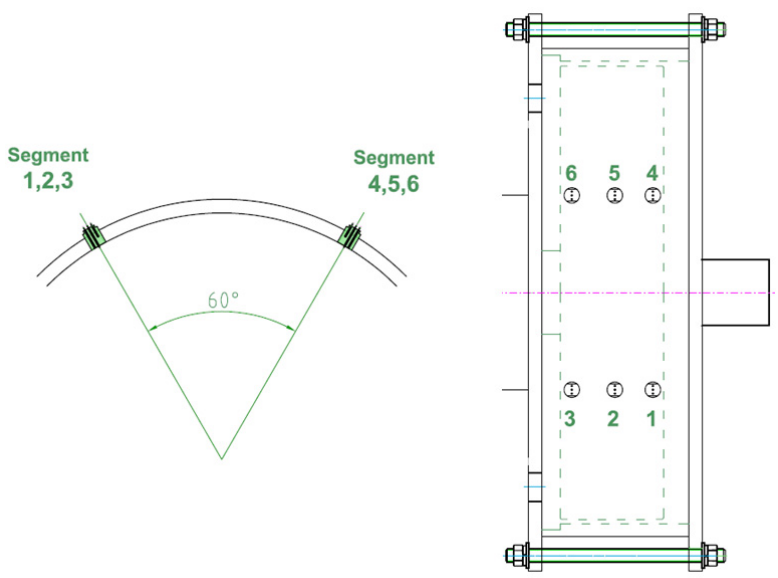

Figure 5. The position of the segments.

\subsection{Experiments set-up}

The simulation of the start-up of steam turbines in this experiment is set up according to time steps listed in Table 1. This setting allows showing the impact of changes in rotor speed on the heat transfer coefficient (HTC). After $70 \mathrm{~min}$ is the nominal rotor speed maintained for $30 \mathrm{~min}$.

Table 1. Rotor speed versus time simulating start-up curve.

\begin{tabular}{|c|c|}
\hline $\begin{array}{c}\text { Time } \\
{[\mathrm{min}]}\end{array}$ & $\begin{array}{c}\text { Rotor speed } \\
\mathrm{rpm} / \mathrm{min}\end{array}$ \\
\hline 0 & 50 \\
\hline 37 & 50 \\
\hline 40 & 2200 \\
\hline 67 & 2200 \\
\hline 70 & 3000 \\
\hline
\end{tabular}

\subsection{Heat transfer}

From the measured gradient of the temperatures $T_{A}$ and $\mathrm{T}_{\mathrm{B}}$ according to a thermal conductivity of the segment, we can determine the heat flux by Equation 1 .

$$
q_{i}=-\lambda \cdot\left(T_{A i}-T_{B i}\right)
$$

The material of segment is stainless steel 316Ti (EN 1.4571). The thermo-physical properties of this steel are determined according to the literature. [9]. Thermal conductivity, density and thermal capacity are approximated by polynomic functions:

$$
\begin{gathered}
\lambda=5 \mathrm{E}-09 \cdot \mathrm{T}^{3}-2 \mathrm{E}-05 \cdot \mathrm{T}^{2}+0,0281 \cdot \mathrm{T}+12,722 \\
\rho=1 \mathrm{E}-07 \cdot \mathrm{T}^{3}-0,0002 \cdot \mathrm{T}^{2}-0,3549 \cdot \mathrm{T}+7958,9 \\
c=-9 \mathrm{E}-14 \cdot \mathrm{T}^{4}+3 \mathrm{E}-10 \cdot \mathrm{T}^{3}-4 \mathrm{E}-07 \cdot \mathrm{T}^{2} \\
\quad+0,0004 \cdot \mathrm{T}+0,4608
\end{gathered}
$$

To calculate heat transfer coefficient (HTC) we use temperature difference between temperature of fluid $T_{C}$ and surface temperature $T_{A}$ together with the heat flux $q_{i}$ we can obtain HTC by:

$$
H T C_{i}=\frac{q_{i}}{\left(T_{C i}-T_{A i}\right)}
$$

For the calculation of the thermo-physical properties of the hot air as the working medium were used recalculation polynomials ASHRAE Standard RP-1485.

\subsection{Data filter}

For data processing and analysis the Diadem software is used. Due to the number of measured data with the noise the filter is used. We used the moving $2^{\text {nd }}$ polynomial filter (Savitzky-Goyal) [10] for a range of values $\mathrm{N}=$ 100.

$$
f\left(y_{i}\right)=\frac{1}{2 \cdot N+1} \cdot \sum_{k=i-N}^{k=i+N} y_{k}, m-N>i>N
$$

\section{RESULTS}

In Figure 6 the temperature at inlet and outlet together with the course of rotor speed is shown. The maximum inlet temperature was $190^{\circ} \mathrm{C}$.

The graph in figure 7 shows the measured temperatures in location $\mathrm{T}_{\mathrm{A}}, \mathrm{T}_{\mathrm{B}}$ and $\mathrm{T}_{\mathrm{C}}$ of the experiment for segments 4, 5 and 6 .

The heat transfer coefficient for each segment is shown in figure 8 . The effect of the increase in rotation speed to heat transfer coefficient is evident in the first speed change from 50 to $2200 \mathrm{rpm}$. During the second speed change from 2200 to $3000 \mathrm{rpm}$ the influence of the increase in heat transfer coefficient is not so distinct. 


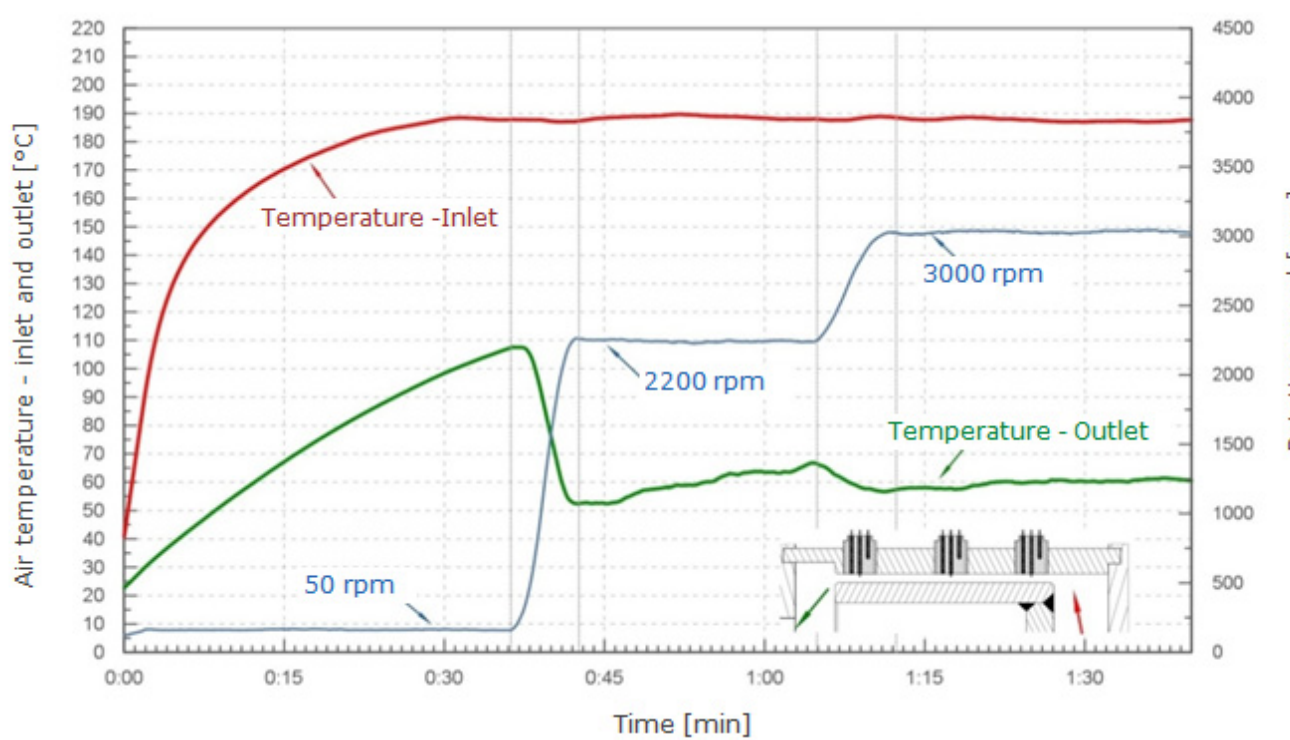

Figure 6. Air temperature - inlet and outlet and rotor speed.

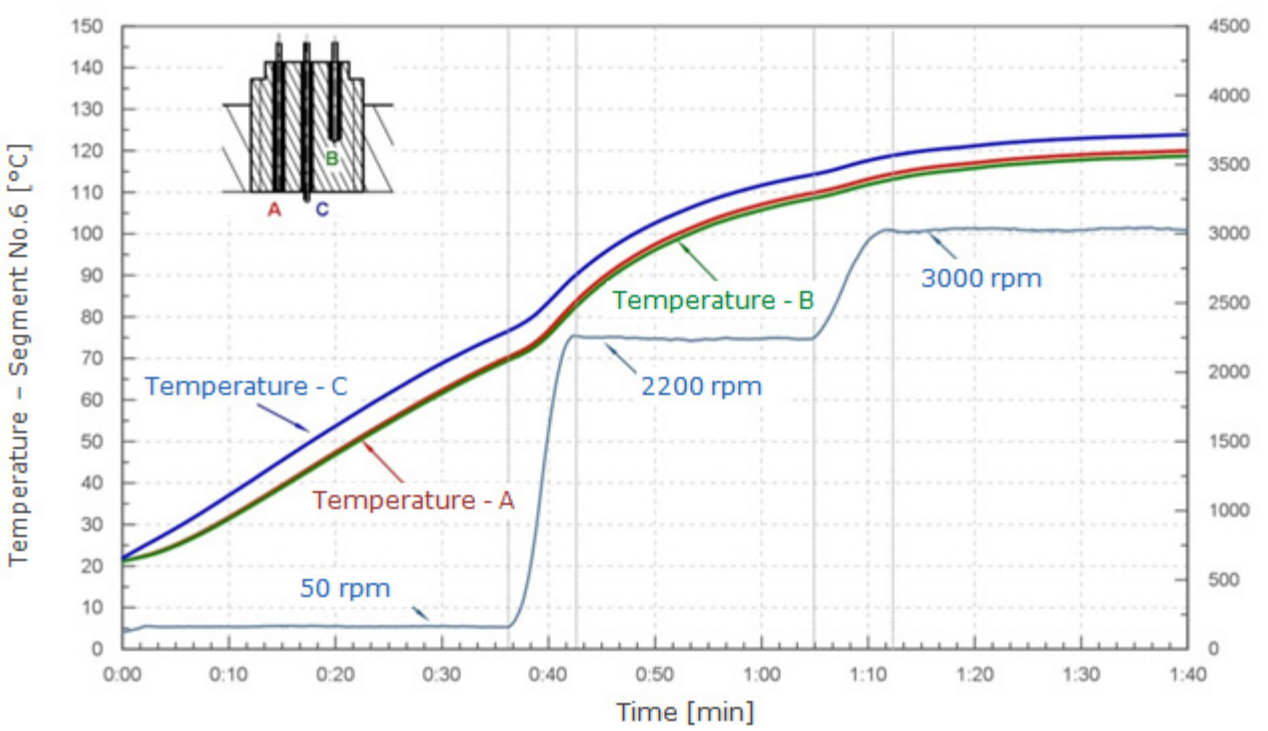

Figure 7. Temperature measured in segment No. 6 .

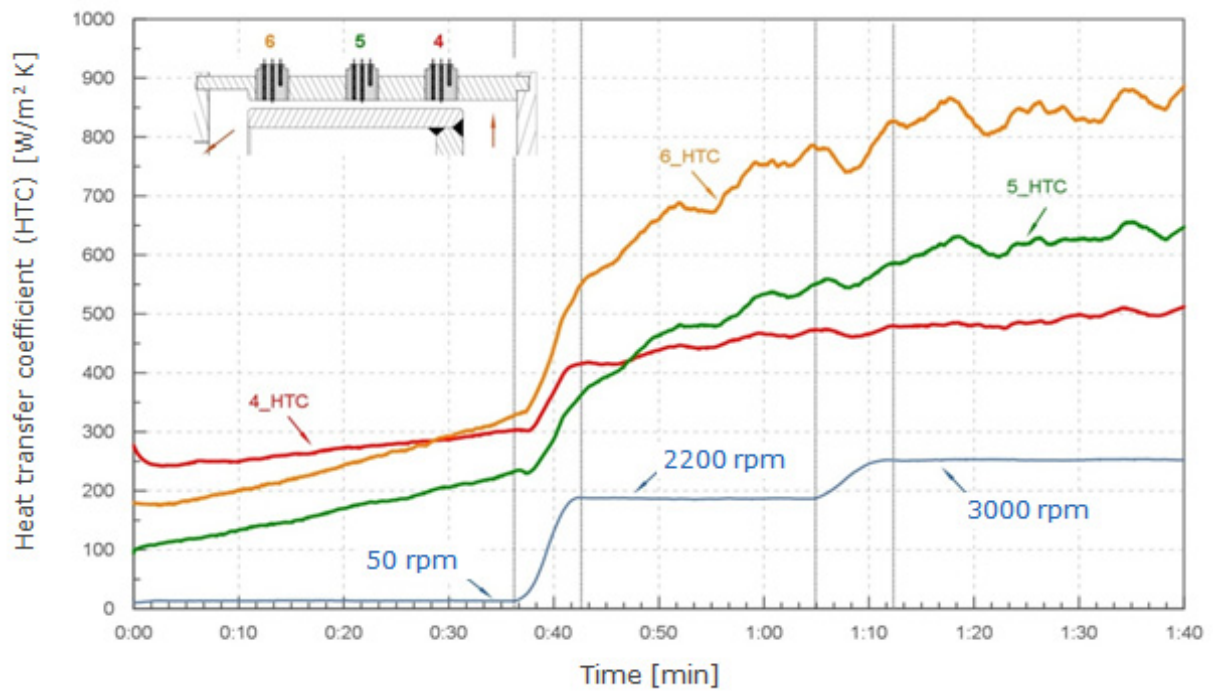

Figure 8. Heat transfer coefficients in segment No. 4,5,6. 


\section{Result discussions}

The Figure 7 shows that the first change of the rotor speed from 50 to $2200 \mathrm{rpm}$ has a significant influence to increasing the Heat transfer coefficient on stator. The second change of rotor speed has not a validate increasing of heat transfer coefficient. In Figure 8 there is shown that the HTC on the first segment (no. 4) is smaller. This can be caused of an axial heat flux throw stator part and also the massive part of rotor opposite of that. To determine this behaviour is necessary to measure temperature distribution of rotor. This can be our future work in this experiment.

\section{Conclusions}

The influence of the changing in rotor speed to the heat transfer coefficient was observed. But mechanism in change of flow vortices in the annular gap must be considered. The segment system of the temperature measuring was stable during the experiment but inverse method for surface temperature measuring must be developed for accurate results.

\section{Acknowledgement}

The authors gratefully acknowledge a financial support from the projects FSI-S-14-2054, ED0002/01/01 NETME CENTRE and NETME CENTRE PLUS (LO1202).

\section{References}

1. E. R. Plotkin, A. Sh. Leiyerovich, I.V Muratova, Thermal Engineering, 18, 41, (1971)

2. T.G. Tret'yakov, Teploenergetika, 18, 27, (1971)

3. P. F. Featherstone, I. Mech. E., U.K. Conference Publication, C189/73, (1973)

4. V.M. Kapinos, J. Eng. Phys, 8, 35, (1965)

5. G. Kosman, M. Rozkozs, Thermal stresses, 6, 389, (2005)

6. W. Kosman, M. Rozozs, K. Nawrat, Applied Thermal Engineering, 29, 3386, (2009)

7. M. Fénot, Z. Bertin, E. Dorignac, G. Lalizel, International Journal of Thermal Sciences, 50, 1138, (2011)

8. Sh. Ch. Tzeng, International Comunications in heat and mass Transfer, 33, 737, (2006)

9. K.C.Mills, Recommended Values of Thermophysical propertiesfor selected comerecial alloys, 244, (2002)

10. A. Savitzky, M. Golay, Analytical Chemistry, 36, 1627 (1964) 